\title{
Clinical evidence for myocardial derecruitment downstream from severe stenosis: pressure-flow control interaction
}

Gianmario Sambuceti, Mario Marzilli, Andrea Mari, Cecilia Marini, Paolo

Marzullo, Roberto Testa, Isabella Raugei, Micaela Papini, Mathis Schluter and

Antonio L'Abbate

Am J Physiol Heart Circ Physiol 279:H2641-H2648, 2000.

You might find this additional info useful...

This article cites 40 articles, 25 of which can be accessed free at:

http://ajpheart.physiology.org/content/279/6/H2641.full.html\#ref-list-1

This article has been cited by 5 other HighWire hosted articles

Paradoxical coronary microcirculatory constriction during ischemia: a synergic function for nitric oxide and endothelin

Claudia Kusmic, Guido Lazzerini, Flavio Coceani, Renata Barsacchi, Antonio L'Abbate and

Gianmario Sambuceti

Am J Physiol Heart Circ Physiol 2006; 291 (4): H1814-H1821.

[Abstract] [Full Text] [PDF]

Coronary microcirculatory vasoconstriction is heterogeneously distributed in acutely ischemic myocardium

Gianmario Sambuceti, Mario Marzilli, Andrea Mari, Cecilia Marini, Mathis Schluter, Roberto

Testa, Michaela Papini, Paolo Marraccini, Giuseppe Ciriello, Paolo Marzullo and Antonio

L'Abbate

Am J Physiol Heart Circ Physiol, May, 2005; 288 (5): H2298-H2305.

[Abstract] [Full Text] [PDF]

Influence of Percutaneous Coronary Intervention on Coronary Microvascular Resistance Index

Bart-Jan Verhoeff, Maria Siebes, Martijn Meuwissen, Bektas Atasever, Michiel Voskuil,

Robbert J. de Winter, Karel T. Koch, Jan G.P. Tijssen, Jos A.E. Spaan and Jan J. Piek

Circulation, January 4, 2005; 111 (1): 76-82.

[Abstract] [Full Text] [PDF]

Paradoxical Increase in Microvascular Resistance During Tachycardia Downstream From a Severe Stenosis in Patients With Coronary Artery Disease : Reversal by Angioplasty Gianmario Sambuceti, Mario Marzilli, Silvio Fedele, Cecilia Marini and Antonio L'Abbate Circulation, May 15, 2001; 103 (19): 2352-2360.

[Abstract] [Full Text] [PDF]

Prologue: new insights into the regulation of the coronary microcirculation William M. Chilian and David D. Gutterman

Am J Physiol Heart Circ Physiol, December 1, 2000; 279 (6): H2585-H2586.

[Full Text] [PDF]

Updated information and services including high resolution figures, can be found at:

http://ajpheart.physiology.org/content/279/6/H2641.full.html

Additional material and information about AJP - Heart and Circulatory Physiology can be found at: http://www.the-aps.org/publications/ajpheart

This information is current as of May 5, 2012.

AJP - Heart and Circulatory Physiology publishes original investigations on the physiology of the heart, blood vessels, and lymphatics, including experimental and theoretical studies of cardiovascular function at all levels of organization ranging from the intact animal to the cellular, subcellular, and molecular levels. It is published 12 times a year (monthly) by the American

Physiological Society, 9650 Rockville Pike, Bethesda MD 20814-3991. Copyright @ 2000 by the American Physiological Society. ISSN: 0363-6135, ESSN: 1522-1539. Visit our website at http://www.the-aps.org/. 


\title{
Clinical evidence for myocardial derecruitment downstream from severe stenosis: pressure-flow control interaction
}

\author{
GIANMARIO SAMBUCETI, ${ }^{1}$ MARIO MARZILLI,${ }^{1}$ ANDREA MARI ${ }^{2}$ CECILIA MARINI, ${ }^{1}$ \\ PAOLO MARZULLO,${ }^{1}$ ROBERTO TESTA,${ }^{1}$ ISABELLA RAUGEI,${ }^{1}$ MICAELA PAPINI, ${ }^{1}$ \\ MATHIS SCHLUTER, ${ }^{1}$ AND ANTONIO L'ABBATE ${ }^{1}$ \\ ${ }^{1}$ Consiglio Nazionale delle Ricerche Institute of Clinical Physiology, Pisa 56100; \\ and ${ }^{2}$ Institute of Systems Science and Biomedical Engineering, Padua 35100, Italy
}

Received 6 June 2000; accepted in final form 23 August 2000

\begin{abstract}
Sambuceti, Gianmario, Mario Marzilli, Andrea Mari, Cecilia Marini, Paolo Marzullo, Roberto Testa, Isabella Raugei, Micaela Papini, Mathis Schluter, and Antonio L'Abbate. Clinical evidence for myocardial derecruitment downstream from severe stenosis: pressure-flow control interaction. Am J Physiol Heart Circ Physiol 279: H2641-H2648, 2000.-To verify the interaction between coronary pressure $(\mathrm{CP})$ and blood flow $(\mathrm{CBF})$ control, we studied nine candidates for angioplasty of an isolated lesion of the left anterior descending coronary artery [i.e., percutaneous transluminal coronary angioplasty (PTCA)]. CBF (i.e., flow velocity $\times$ coronary cross-sectional area at the Doppler tip) and $\mathrm{CP}$ were monitored during washout of $2-5 \mathrm{mCi}$ of ${ }^{133} \mathrm{Xe}$ after bolus injection into the left main artery before and after PTCA. Xe mean transit time (MTT) was calculated as the area under the time-activity curve, acquired by a gamma camera, divided by the dose obtained from a model fit of the Xe curve in the anterior wall. CBF response to intracoronary adenosine $(2 \mathrm{mg})$ was also assessed. PTCA increased baseline CBF (from $14.5 \pm 9.4$ to $20 \pm 8 \mathrm{ml} / \mathrm{min}, P<0.01$ ), coronary flow reserve (from $1.52 \pm 0.24$ to $2.33 \pm 0.8, P<0.01$ ), and $\mathrm{CP}$ (from $64 \pm 9$ to $100 \pm 10 \mathrm{mmHg}, P<0.05$ ). MTT decreased from $89 \pm 32$ to $70 \pm 19 \mathrm{~s}(P<0.05)$ after PTCA; however, MTT and CBF changes were not correlated $(r=$ -0.09 , not significant). Inasmuch as MTT is the ratio of distribution volume to $\mathrm{CBF}$, MTT $\times \mathrm{CBF}$ was used as an index of perfused myocardial volume. Volume increased after PTCA from $23 \pm 18$ to $56 \pm 30 \mathrm{ml}$. A direct correlation was observed between the percent increase in distal $\mathrm{CP}$ and percent increase in perfused volume $(r=0.91, P<0.01)$. Thus low CP was not associated with exhaustion of flow reserve but, rather, with reduction of perfused myocardial volume. These data suggest that, in the presence of a severe coronary stenosis, derecruitment of vascular units occurs that is proportional to the decrease in driving pressure. Residual perfused units maintain a vasomotor tone, thus explaining the paradoxical persistence of coronary reserve.
\end{abstract}

coronary circulation; microcirculation; coronary angioplasty; autoregulation; coronary artery disease

MYOCARDIAL METABOLISM IS STRICTLY aerobic; because of the high oxygen extraction under baseline conditions, increases in oxygen consumption can only be met by

Address for reprint requests and other correspondence: G. Sambuceti, CNR Institute of Clinical Physiology, Via P. Savi 8, 56100 Pisa, Italy (E-mail: battesto@po.ifc.pi.cnr.it). corresponding increases in coronary blood flow $(6,37)$. According to this concept, it is generally assumed that myocardial metabolism is the primary factor controlling microvascular tone and that ischemia implies a maximal vasodilation of coronary microvasculature or exhaustion of coronary flow reserve. However, several experimental studies reported the persistence of a significant vasodilator reserve in moderately ischemic myocardium $(2,7,8,32)$ and, even more, the occurrence of a microvascular constriction during acute severe ischemia $(17,20)$. The mechanisms underlying these phenomena have not been conclusively identified, although they have been attributed to anesthesia (9), an active downregulation of myocardial metabolism below the actual flow availability (7), or a primary microvascular constriction (10). Although this behavior seems paradoxical when the autoregulation of coronary circulation and myogenic reflex are considered, it agrees with observations obtained in peripheral and coronary microcirculation during hemorrhagic shock $(21,31)$ and suggests that further mechanisms, besides oxygen demand, affect vasomotor tone in ischemic myocardium.

The most frequent cause of resting hypoperfusion in humans is the presence of a coronary stenosis. In this setting, a direct correlation exists between coronary blood flow and transstenotic pressure gradient; thus, since resting aortic pressure can be considered relatively constant, an inverse correlation exists between coronary blood flow and distal coronary pressure (18, 19). Several data indicate that the vascular tree, and in particular the microvascular network, is controlled to maintain an adequate pressure at the capillary level to provide correct exchanges between blood and interstitium (42). In the presence of a very severe stenosis, the flow demand might imply an excessive pressure gradient and, thus, an excessively low coronary pressure. Such a vasoconstrictor response to reduced pressure might represent a mechanism able to maintain a correct pressure in the perfused vascular units, provided

The costs of publication of this article were defrayed in part by the payment of page charges. The article must therefore be hereby marked "advertisement" in accordance with 18 U.S.C. Section 1734 solely to indicate this fact. 
that it might act to exclude myocardial units as parallel resistors from perfusion.

The recent introduction of manometer-tipped wires, together with Doppler monitoring of blood flow, allows the measurement of microvascular coronary resistance in patients with coronary artery disease. When coupled with the analysis of the washout of a freely diffusible tracer and applied to the clinical model of coronary angioplasty, this technology permits the study of coronary blood flow and perfused myocardial volume at different coronary pressures under stable systemic hemodynamics. This possibility is crucial, since the stability of aortic pressure minimizes the triggering of reflexes that might directly affect coronary vasomotor tone. We used this experimental setting to estimate the interrelationship between coronary pressure, blood flow, and myocardial volume.

\section{MATERIALS AND METHODS}

Study population. Nine candidates for coronary angioplasty (mean age $61 \pm 3 \mathrm{yr}$ ) were included according to the following criteria: 1) history of stable angina, 2) electrocardiographic (ECG) evidence of exercise-induced ischemia, 3) single-vessel disease of the left anterior descending coronary artery, 4) no previous myocardial infarction, 5) left main stem longer than $2 \mathrm{~cm}, 6$ ) no angiographic evidence of collateral circulation, 7) absence of arterial hypertension and/or left ventricular hypertrophy, and 8) no diabetes.

Study protocol. Patients were studied after overnight fast, under active treatment with oral diltiazem (60 mg 3 times/ day), isosorbide mononitrate ( $20 \mathrm{mg} 3$ times/day), and aspirin. An 8.0-F guiding catheter was advanced into the left main coronary artery, a control angiography was performed, and the best projection was selected. Heparin (10,000 IU) was injected intravenously; isosorbide dinitrate $(0.6 \mathrm{mg})$ was administered into the heart. A 0.014-in. fiber-optic pressuremonitoring guide wire (Radi Medical, Uppsala, Sweden) was calibrated and positioned distal to the stenosis (34). Finally, a 2.5-F Doppler-tipped catheter (Millar Instruments, Houston, TX) was placed in the prestenotic segment, and a coronary angiography was again obtained to measure cross-sectional area at the tip of the Doppler catheter. Care was taken to avoid side branching between the catheter tip and the stenosis and to maintain the catheter in the center of the lumen to obtain a stable flow-velocity signal.

A small field-of-view mobile gamma camera (model F1, Elscint, Haifa, Israel) was brought to the catheterization room. The system was equipped with a low-energy, highsensitivity parallel-hole collimator and was oriented on the patient's chest in a $70^{\circ}$ left anterior oblique projection.

Stable blood flow and hemodynamics were verified for $\geq 5$ min before baseline recordings. Thereafter, a bolus of $2-4$ $\mathrm{mCi}$ of ${ }^{133} \mathrm{Xe}$ was rapidly injected through the guiding catheter soon after initiation of a dynamic acquisition set according to the following parameters: energy window centered on the photo peak of ${ }^{133} \mathrm{Xe}$ and one frame every $2 \mathrm{~s}$ for $180 \mathrm{~s}$. At the end of acquisition, a bolus of adenosine $(2 \mathrm{mg})$ was selectively injected into the left anterior descending coronary artery through the Doppler catheter.

The following signals were continuously monitored: 1) four ECG leads (I, II, III, and V4), 2) phasic and mean aortic pressure, 3) phasic and mean distal coronary pressure, and 4) phasic and mean coronary blood flow velocity. Paper recordings $(2.5 \mathrm{~cm} / \mathrm{s})$ were obtained at baseline and $30 \mathrm{~s}$ after intracoronary adenosine. Distal coronary pressure was also measured during balloon coronary occlusion.

After completion of this protocol, the Doppler catheter was removed, and all patients underwent coronary angioplasty. After revascularization, the Doppler catheter was advanced in the treated vessel, and the protocol was repeated as before angioplasty.

Data analysis. Stenosis severity (percent lumen area reduction) and vessel diameter at the tip of the Doppler catheter were measured using an automated edge detection system (Mipron, Kontron). Coronary blood flow index was obtained by multiplying mean blood flow velocity by crosssectional area at the site of the Doppler transducer, as previously described (16).

Mean transit time of Xe was calculated according to the residue detection method. Original images were grouped into 20-s frames to allow a better definition of the left ventricular walls. Two regions of interest were drawn on the anterior and posterolateral wall, and frames were displayed in cine mode to verify a stable geometry. Thereafter, the computer was asked to plot the time-activity curves in stenotic-anterior and control-posterolateral regions using the original 2-s frames.

The mean transit time was calculated, according to the stochastic analysis, from the tracer residue curves as the ratio of the area under the curve from zero to infinity to the dose entering the region (44). These two values were estimated using the model described below.

The tracer mass balance in the region of interest is

$$
q=f_{\text {in }}-f_{\text {out }}
$$

where $\mathrm{q}$ is the tracer mass in the region, which is proportional to the Xe radioactivity, $f_{\text {in }}$ is influx, and $f_{\text {out }}$ is efflux. For the influx, the following representation was used

$$
\mathrm{f}_{\mathrm{in}}(t)=\mathrm{D} \Gamma(\theta)(\mu / \theta)^{\theta} t^{\theta-1} e^{-(\theta / \mu) t}
$$

where $\mathrm{D}$ is the tracer dose entering the region (the integral from zero to infinity of $f_{\text {in }}$ ), $\Gamma$ is the gamma function, $\mu$ is the mean transit time of $f_{i n}$, which is related to the delay of the tracer appearance into the region, and $\theta$ quantifies the dispersion of the dose around its mean transit time $\mu$. Equation 2 , with only three parameters, ensures a flexible description of the input function, which starts from zero, rapidly reaches a peak, and returns to zero. Equation 2 is actually equivalent to $\mathrm{f}_{\text {in }}=a t^{b} e^{-c t}$, where $a, b$, and $c$ are the parameters, but the more complex expression is used because the parameters have a clear meaning.

The efflux is represented as the convolution of the influx and the transit time density function of the region. The transit time density function, $p(t)$, was modeled as the convolution of a single-exponential function with a two-exponential function. This gives $p(t)$ the characteristics of a typical efflux curve, which rapidly rises to a peak and decays with a biphasic pattern. The expression used for $p(t)$ is

$$
p(t)=\beta e^{-\beta t} \otimes\left[w \alpha_{1} e^{-\alpha_{1} t}+(1-w) \alpha_{2} e^{-\alpha_{2} t}\right]
$$

where $\otimes$ is the convolution operator. The parameter $\beta$ determines the initial rising of $p(t), \alpha_{1}$ and $\alpha_{2}$ are the exponents of the biphasic decay, and $w$ represents the relative contribution of the first exponential term of the decay.

By combining Eqs. 1-3, the differential equation describing the tracer residue curves is obtained. This equation was solved using inversion of Laplace transforms, inasmuch as Eqs. 1-3 can be conveniently handled in the domain of Laplace transforms. Inversion of Laplace transforms was performed with the algorithm of de Hoog et al. (12). The parameters of Eqs. 1 and 2 were estimated by least-squares 
fit of the tracer residue curves. Calculations were performed using the language of technical computing MatLab (the routine for the inversion of Laplace transforms was kindly provided by Karl Hollenbeck, Dept. of Hydrodynamics and Water Resources, Technical University of Denmark, Lyngby, Denmark).

The mean transit time in the region (calculated as area/ dose) is equal to the mean transit time of $p(t)$, which is obtained from the parameters of $E q$. 3. Thus, although the model provides an estimate of the input function (Eq. 2) and of the transit time density function of the region $(E q .3)$, it is in practice only used for estimating the dose and for extrapolating the tail of the residue curve beyond the observation window.

Mean transit time of a diffusible tracer is the ratio of the distribution volume of the tracer to the flow crossing that volume. When ${ }^{133} \mathrm{Xe}$ is injected into the left main coronary artery, such a formulation can be written as follows

$$
\mathrm{MTT}=\mathrm{V} / \mathrm{CBF}
$$

where MTT is the mean transit time, $\mathrm{V}$ is the distribution volume of $\mathrm{Xe}$, and $\mathrm{CBF}$ is the blood flow in the labeled volume. Accordingly, the distribution volume of Xe, which is an index of the perfused myocardium, was computed by rearranging $E q .4$ as follows

$$
\mathrm{V}=\mathrm{MTT} * \mathrm{CBF}
$$

Statistical analysis. Values are means \pm SD. ANOVA, followed by Newman-Keuls procedure for multiple comparisons and repeated measures, was used in each population to identify significant changes in blood flow indexes at the various stages of the protocol before or after angioplasty. Linear regression analysis was performed by least-squares method. $P<0.05$ was considered significant.

\section{RESULTS}

Clinical and hemodynamic findings. No serious side effects occurred during the study. Left ventriculography showed mild to moderate anterior hypokinesis in three of nine patients; left ventricular ejection fraction was $0.56 \pm 0.04$. Left ventricular end-diastolic pressure was $9 \pm 4 \mathrm{mmHg}$. Coronary angioplasty was successful in all patients and was optimized by a stent deployment in seven of nine patients. Percent arterial area reduction decreased from $96 \pm 4$ to $14 \pm 6 \%(P<$ 0.01). After revascularization, there were no significant changes in heart rate or systolic or diastolic aortic pressure (Table 1).

Coronary pressure and Doppler blood flow index. Baseline blood flow index was $14.5 \pm 9.4 \mathrm{ml} / \mathrm{min}$ and increased in all patients to $152 \pm 24 \%$ (range $115-$ $200 \%, P<0.02)$ after adenosine. Transstenotic pres- sure gradient was $27 \pm 14 \mathrm{mmHg}$ at baseline, increased to $37 \pm 15 \mathrm{mmHg}$ after adenosine $(P<0.01)$, and virtually disappeared after revascularization at rest ( $2 \pm 2 \mathrm{mmHg}$ ) and after adenosine $(5 \pm 4 \mathrm{mmHg}$, $P<0.01$ vs. corresponding values before revascularization). Mean distal pressure was $64 \pm 9 \mathrm{mmHg}$ at baseline, decreased to $54 \pm 10 \mathrm{mmHg}$ after adenosine $(P<0.01$ vs. baseline), and markedly $(P<0.01)$ increased after angioplasty at rest $(100 \pm 10 \mathrm{mmHg})$ and after adenosine (99 $\pm 9 \mathrm{mmHg}$; Table 1$)$. During balloon coronary occlusion, coronary wedge pressure was $19 \pm 7 \mathrm{mmHg}$.

Angioplasty increased $(P<0.01)$ blood flow index to $20 \pm 8 \mathrm{ml} / \mathrm{min}$, corresponding to $180 \pm 93 \%$ of baseline (Figs. 1 and 2). Similarly, a large increase was observed in maximal flow capacity, which increased from $152 \pm 24$ to $324 \pm 242 \%(P<0.01)$ of preangioplasty baseline flow, and coronary flow reserve, which increased from $1.52 \pm 0.24$ to $2.33 \pm 0.8(P<0.01)$.

Xe washout analysis. Background activity, before postpercutaneous transluminal coronary angioplasty study, was virtually absent in all cases, resulting in $<1 \%$ of peak activity after tracer injection. Similarly, heart-to-background ratio was $>4$ at the end of all studies. Fitting of the Xe washout curve was possible in all cases. The model residuals, i.e., the differences between the measured and model-predicted Xe radioactivity, indicated that the model fit was acceptable. In the majority of the time points from 0 to $180 \mathrm{~s}(81 \%$ of the points), the mean residuals were within $2 \mathrm{SE}$ for the point, indicating no bias. Although in a significant proportion of the time points $(19 \%)$ the mean residuals differed from zero by $>2 \mathrm{SE}$, the value was $<1 \%$ of the peak value, and the mean residuals exhibited small oscillations but not a tendency to under- or overestimate the slope of the tracer curves. These results indicate that the model extrapolation of the tail of the tracer curve was adequate. The model-estimated dose entering the regions of interest was $113 \pm 1.3 \%$ (SE) of the measured Xe peak (all 18 curves pooled). This result is consistent with the notion that a small fraction of the tracer dose ( $13 \%$ in our calculation) leaves the region of interest before the peak value is reached.

Mean transit time of Xe washout was $89 \pm 32 \mathrm{~s}$ at baseline and decreased to $70 \pm 19 \mathrm{~s}(76 \pm 21 \%, P<$ 0.05 ) after angioplasty. In the contralateral region, this variable similarly decreased from $94 \pm 34$ to $68 \pm 24 \mathrm{~s}$ $(P<0.05)$. Thus mean transit time was similar in the stenotic and remote myocardium before and after re-

\begin{tabular}{|c|c|c|c|c|}
\hline & \multicolumn{2}{|c|}{ Before Angioplasty } & \multicolumn{2}{|c|}{ After Angioplasty } \\
\hline & Baseline & Intracoronary adenosine & Baseline & Intracoronary adenosine \\
\hline Heart rate, beats/min & $70 \pm 10$ & $73 \pm 10$ & $70 \pm 8$ & $72 \pm 9$ \\
\hline Mean aortic pressure, $\mathrm{mmHg}$ & $97 \pm 15$ & $101 \pm 10$ & $100 \pm 9$ & $104 \pm 9$ \\
\hline Mean coronary pressure, $\mathrm{mmHg}$ & $64 \pm 9$ & $54 \pm 10^{*}$ & $100 \pm 10^{* \dagger}$ & $99 \pm 9 * \dagger$ \\
\hline
\end{tabular}

Table 1. Hemodynamic data before and after revascularization

Values are means \pm SD. Heart rate and aortic pressure did not change throughout the study. $* P<0.05$ vs. baseline before percutaneous transluminal coronary angioplasty (PTCA); $\dagger P<0.05$ vs. intracoronary adenosine before PTCA. 
Before

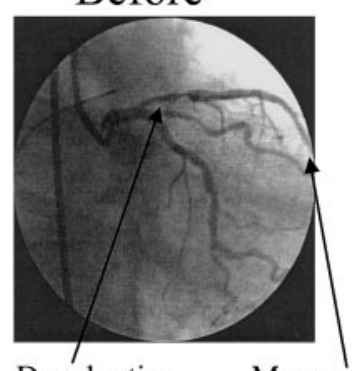

Doppler tip Manometer tip

Fig. 1. Coronary angiography (top), pressure and flow velocity traces (middle), and Xe washout curves (bottom) at rest and before and after percutaneous transluminal coronary angioplasty (PTCA). PTCA abolished epicardial stenosis and increased distal coronary pressure (dCP) to values similar to aortic pressure (AoP) at rest and after intracoronary adenosine. Adenosine significantly increased coronary blood flow velocity (CBFV) before and after PTCA. Balloon coronary occlusion markedly decreased dCP. PTCA markedly increased coronary blood flow at rest and after adenosine. Nevertheless, Xe washout curve did not change after revascularization in the jeopardized area (solid line) and remained similar to the washout curve observed in the remote region (dashed line). Thus the increase in flow induced by PTCA was not associated with a marked increase in Xe mean transit time. ECG, electrocardiogram.
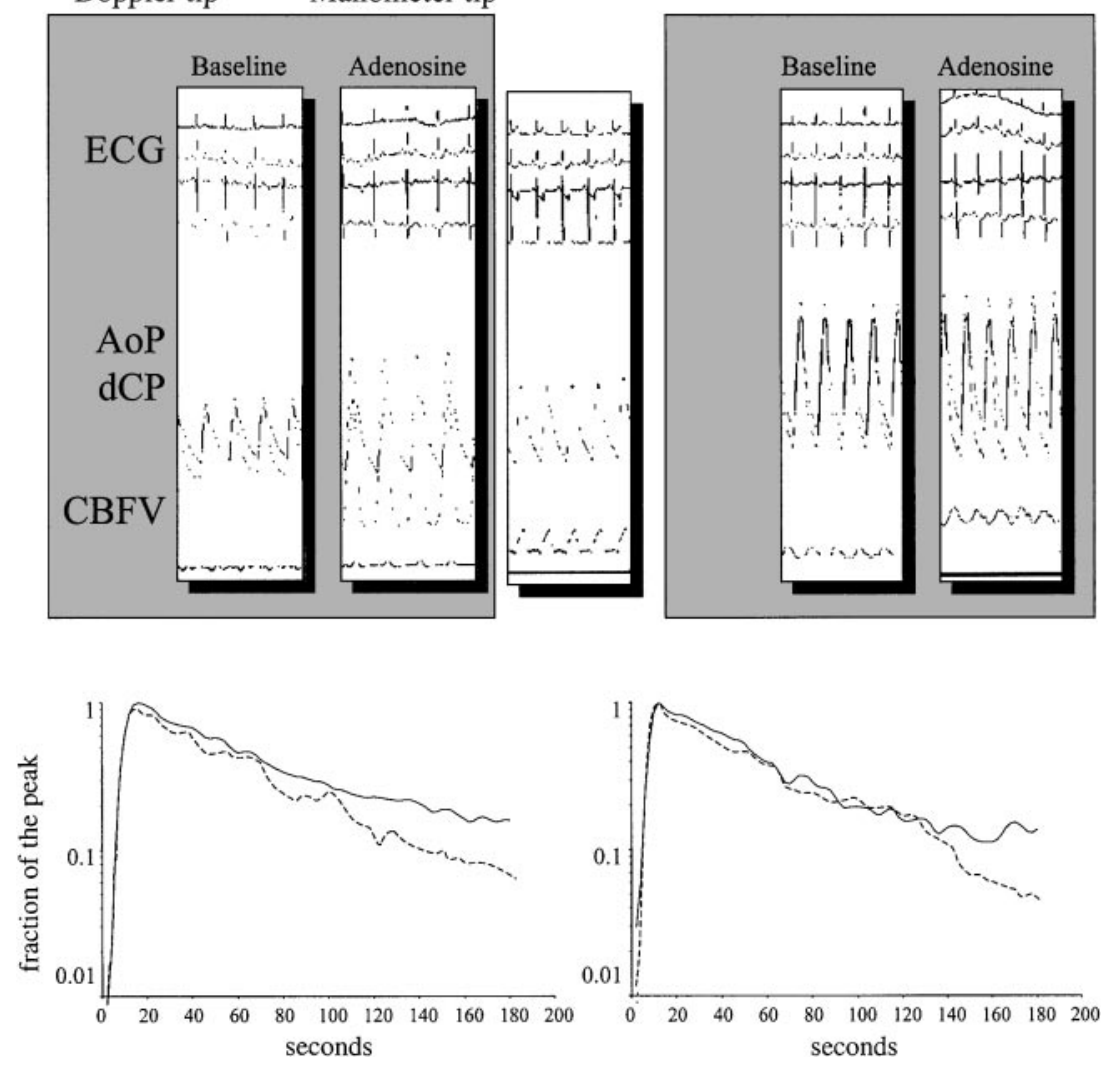

vascularization. No correlation was observed between the increase in coronary blood flow and the decrease in mean transit time of $\mathrm{Xe}$ induced by revascularization $(r=0.19$, not significant; Fig. 3).

The volume of distribution of Xe, derived by Doppler coronary blood flow $\times$ mean transit time, increased from $23 \pm 18$ to $56 \pm 30 \mathrm{ml}(P<0.05)$ after revascularization; this phenomenon showed a large variation between patients (Fig. 4); however, a close direct correlation was observed between the increase in driving coronary pressure and the increase in volume of distribution of the tracer $(r=0.91, P<0.01$; Fig. 5$)$. No correlation was observed between changes in perfused myocardial volume and coronary wedge pressure measured during balloon coronary occlusion $(r=0.13$, not significant).

\section{DISCUSSION}

In the present study, angioplasty of severe coronary artery stenosis was associated with a marked increase in absolute coronary blood flow and coronary flow reserve assessed by intracoronary Doppler catheter. However, the increase in resting coronary blood flow was markedly larger than (and not correlated with) the decrease in mean transit time of ${ }^{133} \mathrm{Xe}$, indicating a significant increase in the volume of distribution of the tracer and, thus, in the mass of perfused myocardium after revascularization. Moreover, a significant correlation was observed between the increase in the perfused volume and the increase in distal coronary pressure. These findings suggest that, in the presence of a very severe stenosis and transstenotic pressure gradient, observed resting coronary blood flow is distributed to only a portion of the vascular units that are perfused in the absence of the stenosis, i.e., after revascularization. The spatial and temporal distribution of the perfused units, within the jeopardized region, cannot be identified from the present study.

Coronary angioplasty and coronary blood flow. Angioplasty markedly increased Doppler coronary blood 
A

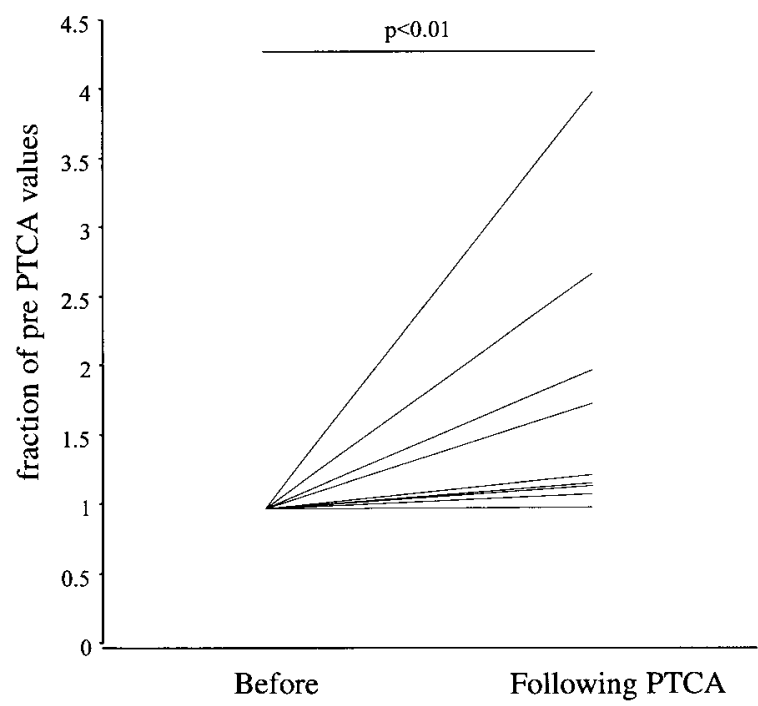

B

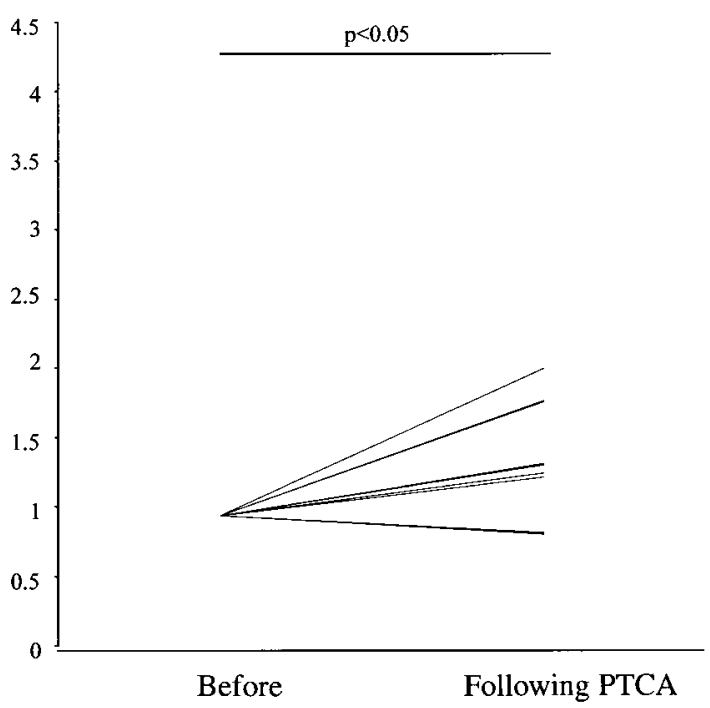

Fig. 2. A: changes in absolute coronary blood flow $(\mathrm{CBF})$ (blood flow velocity $\times$ cross-sectional area of the coronary artery) induced by PTCA. B: effect of PTCA on specific flow (flow per unit mass) as measured by the inverse of Xe mean transit time (MTT). The increase in absolute flow was higher than the increase in specific myocardial flow.

flow index in all patients. Such a phenomenon might reflect the occurrence of postocclusion reactive hyperemia (39) or the presence of a significant hypoperfusion before vessel recanalization. After revascularization, adenosine markedly increased coronary blood flow, revealing the presence of a significant coronary flow reserve. In agreement with previous studies (4, 33, 40), this observation strongly suggests a chronic hypoperfusion in myocardial regions exposed to a reduced driving pressure due to the presence of a severe stenosis. However, Xe mean transit time was remarkably similar in the myocardium supplied by the stenotic artery and in the remote region before and after angioplasty, despite the marked increase in flow to the revascularized myocardium. Since, according to tracer kinetic theory, Xe mean transit time is the ratio of flow to tracer distribution volume $(23,30,44)$, this observation would imply a similar increase in blood flow to both regions or an increase in the distribution volume in the

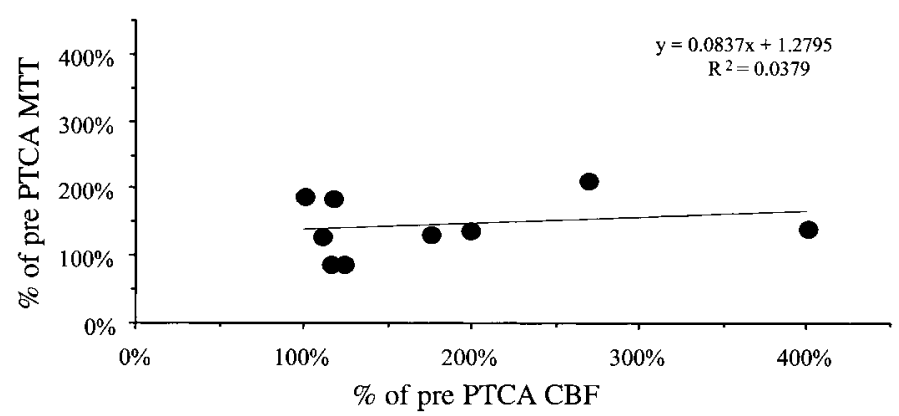

Fig. 3. Relationship between changes in absolute CBF (blood flow velocity $\times$ cross-sectional area of the coronary artery) and changes in specific myocardial blood flow [inverse of Xe mean transit time (MTT)]. In a homogeneously perfused system with a constant volume, a linear direct correlation should be observed between these 2 variables. The lack of this observation suggests an effect of revascularization on the distribution volume of the tracer. revascularized myocardium. Coronary blood flow in the nonstenotic coronary artery was not measured. However, heart rate and systolic aortic pressure remained unchanged, and thus, in agreement with previous studies, an increase in blood flow in this region, beyond the modest increase in flow compatible with the increase in Xe washout rate, seems unlikely (33). By contrast, at least two observations suggest that the volume of distribution of $\mathrm{Xe}$ in the revascularized area was actually increased by angioplasty: the increase in flow was higher than (and not correlated with) the decrease in mean transit time, and the increase in tracer distribution volume was directly correlated with the increase in driving coronary pressure. The volume of distribution of Xe reflects two main factors: the so-called partition coefficient (related to the chemical tissue constituents) (11) and the perfused myocardial mass (related to the mass of the myocardium reached by the tracer and thus by flow) $(23,30)$. Since dramatic changes in the chemical components of the revascular-

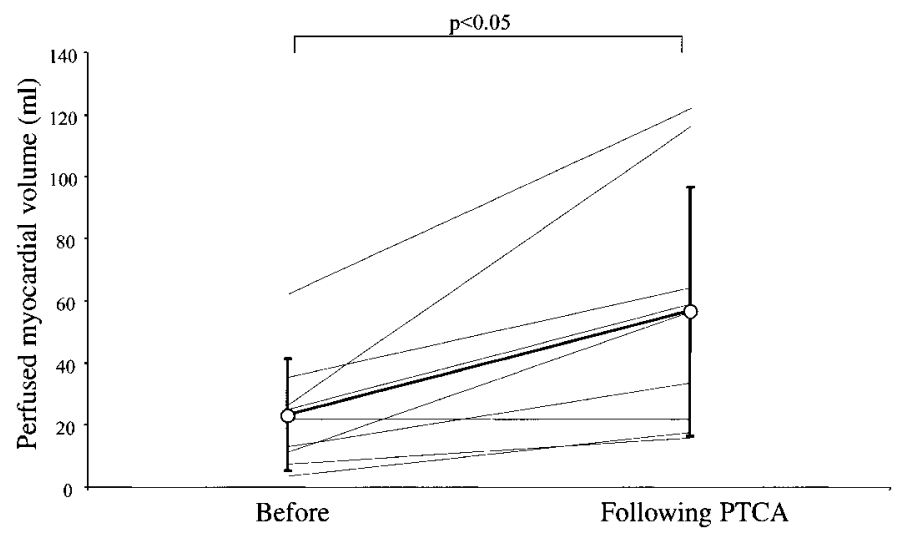

Fig. 4. Effect of PTCA on perfused myocardial volume $(\mathrm{CBF} \times \mathrm{Xe}$ MTT) before and after PTCA. 


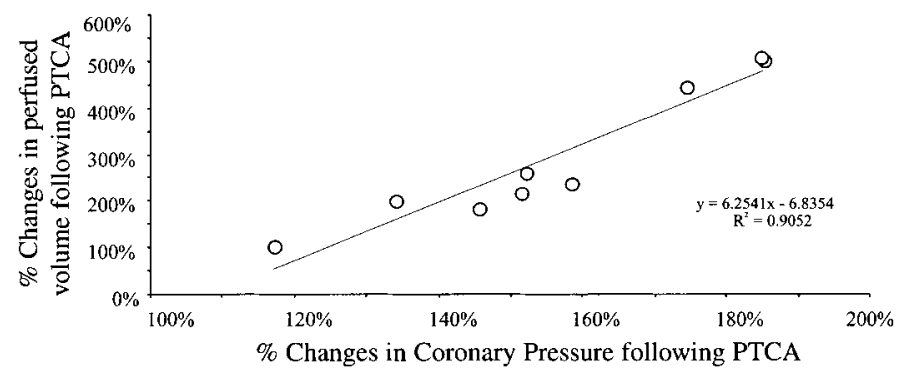

Fig. 5. Relationship between changes in coronary pressure and changes in perfused myocardial volume $(\mathrm{CBF} \times \mathrm{Xe} \mathrm{MTT})$ induced by revascularization (PTCA). A close direct correlation was observed between these 2 variables, indicating an effect of driving coronary pressure on the mass of myocardium perfused.

ized myocardium are unlikely to occur in such a short period of time, it seems reasonable to hypothesize an increase in perfused myocardial mass. Thus these findings strongly suggest a relationship between driving coronary pressure and amount of the perfused myocardium in patients with coronary artery disease.

Relationship between metabolic control of blood flow and vascular control of pressure. An increase in flow heterogeneity during ischemia has been described previously $(13,31,41,43,45)$. This phenomenon has been frequently explained by a heterogeneous distribution of extravascular compressing forces or by the structure of the coronary tree $(3,24)$. Actually, it is well known that such a difference might explain the relative reduction in subendocardial flow during maximal vasodilation, which has been documented in regions supplied by a severely stenotic vessel or at low pressure $(22,24)$. However, in the present study, resting hypoperfusion was not associated with an exhausted vasodilator reserve, inasmuch as adenosine increased flow across the coronary microcirculation, despite a reduced distal coronary pressure, indicating the persistence of a residual microcirculatory vasomotor tone. In line with this finding, several authors documented vasodilator reserve in the hypoperfused myocardium $(2,7,8,19,32)$, and, more recently, we observed even an active, severe vasoconstriction of coronary microvasculature during unstable angina (29) and ischemia induced by atrial pacing tachycardia (38). This mismatch between myocardial metabolism and microvascular resistance behavior might appear paradoxical when myocardial metabolism is assumed to be the primary determinant of vasomotor tone regulation. However, one should also consider the relevance of pressure-driven control, which avoids the notion that changes in aortic pressure are directly brought at the capillary level with the obvious dramatic consequence of altered plasma-interstitial water and solute exchanges $(26,42)$. The myogenic reflex, which is the most acknowledged mechanism in coronary autoregulation, could operate such a control $(5,15)$. In patients with coronary artery disease, it seems conceivable that the putative vasodilation required to match oxygen demand with blood flow would imply an excessive pressure drop across a severe stenosis (19). Since resting aortic pressure can be con- sidered relatively stable, the pressure gradient would result in an excessively low capillary pressure, thus hampering the Starling forces $(26,42)$. Under these conditions, the only mechanisms able to preserve capillary pressure would be a venous constriction or the exclusion of perfused units in a parallel circuit model. In the present study, perfused myocardial volume increased after revascularization, and this increase was directly correlated with the increase in driving coronary pressure. This observation suggests that the transstenotic pressure gradient caused a reduction in perfused myocardium and a "derecruitment" of vascular units from perfusion. These data do not elucidate whether this is an all-or-none phenomenon nor do they verify whether the exclusion of any given vascular unit is continuous or intermittent. However, in agreement with data by Chilian and Layne (10), these findings suggest that the coronary microvasculature reacts to a markedly reduced pressure by an active vasoconstriction. Such a response leads to exclusion of myocardial tissue from perfusion and thus to restoration of adequate flow rates and microvascular pressures in the perfused units. Such a mechanism might be even more powerful than the effects of myocardial metabolism on overall microvascular resistance. This concept closely agrees with the experimental evidence of a recruitment in perfused myocardial tissue in response to increases in coronary pressure (25) under autoregulation and during maximal vasodilation. Similarly, this model might also help explain the marked heterogeneity of blood flow, coronary reserve, and metabolic indexes of ischemia, which has been observed in the experimental setting $(1,13,21,27,31,41,45)$.

Clinical implications. In patients with coronary artery disease, myocardial dysfunction is often observed in regions subtended by severely stenotic coronary arteries. In several instances, a contractile recovery can be observed after revascularization (4). Since this phenomenon has important prognostic implications, its mechanisms have been extensively investigated. It has not been fully defined whether regional dysfunction is the effect of chronic hypoperfusion (myocardial hibernation) (35) or prolonged dysfunction after repetitive ischemia (myocardial stunning) (28). On the one hand, studies evaluating myocardial perfusion by the delivery or uptake of tracers, such as microspheres, ${ }^{201} \mathrm{Tl}-\mathrm{S}$ estamibi, or $\left[{ }^{13} \mathrm{~N}\right]$ ammonia, documented resting perfusion defects in viable myocardial regions (4). In contrast, this finding has been rarely reported by positron emission tomography studies of myocardial blood flow with radioactive water as a flow tracer (28). Similarly, although the former techniques often reported an increase in blood flow to revascularized areas, water studies of blood flow showed a smaller effect of revascularization on resting myocardial blood flow. These puzzling discordances might reflect the different kinetics between diffusible tracers (which estimate blood flow washout rates) and deposit tracers (which estimate blood flow by the amount of tracer delivered) $(14,23,43)$. In the present study, mean transit time of Xe was not markedly reduced in the "ischemic" region, 
indicating a near-to-normal specific flow in the perfused myocardium. In agreement with this finding, Xe mean transit time did not markedly decrease after revascularization However, the mass of the perfused myocardium was reduced, since it markedly increased after the restoration of a normal driving pressure. This finding indicates that a significant fraction of the ischemic myocardium was excluded from perfusion and thus from tracer handling before revascularization. Accordingly, it seems conceivable that, at least in some instances, diffusible tracers might overestimate myocardial blood flow because of underestimation of myocardial mass, whereas they could underestimate the improvement in blood flow induced by revascularization because of the increase in perfused mass induced by the increase in driving coronary pressure.

Finally, these data point out the relevance of perfusion pressure as a primary determinant of the regulation of myocardial blood flow in the myocardium supplied by a severely stenotic coronary artery under resting conditions. The early beneficial effect of revascularization on blood flow regulation might reflect the increase in driving coronary pressure. Actually, this mechanism might also retain a potential relevance in the precipitation of acute ischemia, thus justifying the finding of a paradoxical vasoconstrictor response to increasing myocardial metabolic demand (38). Methods able to verify such a possibility might help more accurately characterize the pathogenesis of ischemia in patients with coronary artery disease.

Limitations. The increase in coronary blood flow observed after angioplasty might reflect the disappearance of collateral circulation. In this instance, actual perfusion rate to the myocardium might have been less modified by coronary angioplasty, thus causing the relative reduction in mean transit time. However, patients with angiographic evidence of collaterals were excluded from the present study. Moreover, coronary wedge pressure was remarkably low in all patients, and no correlation was observed between this variable and changes in coronary blood flow and perfused volume. Coronary pressure measured during balloon occlusion is considered the most accurate clinical descriptor of collateral feeding to the ischemic vascular bed (36). Thus these observations imply that collaterals were not extensively developed in these patients and that the observed increase in coronary blood flow was not closely related to their presence.

Xe washout was not monitored after adenosine administration because of the problems in maintaining stable levels of coronary blood flow, coronary pressure, and heart rate during adenosine infusion for the duration of Xe washout. Accordingly, the present study does not elucidate the behavior of perfused myocardial volume under pharmacological vasodilation and at low perfusion pressure. An increase in perfused mass, under vasodilation, might have further confirmed the hypothesis of the active nature of the heterogeneous vasoconstriction.

Coronary blood flow was not monitored in the contralateral region. This evaluation might have allowed a more precise definition of perfused volume in a myocardium not subjected to changes in perfusion pressure, at least in the large arterial system. However, placing a further instrument in an untreated coronary artery was considered unethical in patients with coronary artery diseases underlying a study during coronary angioplasty.

Utilization of the gamma-camera allowed accurate myocardial imaging, with clear separation between regions supplied by the stenotic or nonstenotic vessels. However, an exact definition of possible regional differences within the region supplied by the left anterior descending coronary artery could not be identified with the planar imaging method adopted. Moreover, because of the limited spatial resolution of the method, no conclusion can be drawn about the distribution of flow heterogeneity or the individual size of hypoperfused or normally perfused myocardial units. These limitations, together with the limited patient population, prevent an accurate definition of the shape of the relationship between coronary pressure and perfused myocardial mass as well as the temporal variability of myocardial perfusion.

\section{REFERENCES}

1. Austin RE, Aldea GS, Coggins DL, Flynn AE, and Hoffman JIE. Profound spatial heterogeneity of coronary reserve. Circ Res 67: 319-331, 1990.

2. Aversano T and Becker LC. Persistence of coronary vasodilator reserve despite functionally significant flow reduction. $A m \mathrm{~J}$ Physiol Heart Circ Physiol 248: H403-H411, 1985.

3. Bassingthwaighte JB, King RB, and Roger SA. Fractal nature of regional blood flow heterogeneity. Circ Res 65: 578590,1989

4. Bax JJ, Wijns W, Cornel JH, Visser FC, Boersma E, and Fioretti PM. Accuracy of currently available techniques for prediction of functional recovery after revascularization in patients with left ventricular dysfunction due to chronic coronary artery disease: comparison of pooled data. J Am Coll Cardiol 30: 1451-1460, 1997.

5. Bayliss WM. On the local reaction of the arterial wall to changes of internal pressure. J Physiol (Lond) 28: 220-231, 1902.

6. Belloni FL and Sparks HV. Dynamics of myocardial oxygen consumption and coronary vascular resistance. Am J Physiol Heart Circ Physiol 233: H34-H43, 1977.

7. Bristow JD, Arai AE, Anselone CG, and Pantely GA. Response to myocardial ischemia as a regulated process. Circulation 84: 2580-2587, 1991.

8. Canty JM and Klocke FJ. Reduced regional myocardial perfusion in the presence of pharmacologic vasodilator reserve. Circulation 71: 370-371, 1985.

9. Canty JM and Smith TP. Adenosine-recruitable flow reserve is absent during myocardial ischemia in unanaesthetised dogs studied in the basal state. Circ Res 76: 1079-1087, 1995.

10. Chilian WM and Layne SM. Coronary microvascular responses to reductions in perfusion pressure: evidence for persistent arteriolar vasomotor tone during coronary hypoperfusion. Circ Res 66: 1227-1238, 1990.

11. Conn HL. Equilibrium distribution of radioxenon in tissue. Xenon-hemoglobin association curve. J Appl Physiol 16: 10651070, 1961

12. De Hoog FR, Knight JH, and Stokes AN. An improved method for numerical inversion of Laplace transforms. SIAM $J$ Sci Stat Comput 3: 357-366, 1982.

13. Factor SM, Sonnenblick EH, and Kirk ES. The histologic border zone of acute myocardial infarction-islands or peninsulas? Am J Pathol 92: 111-124, 1978.

14. Fluck DS, Etherington PJE, O'Hare D, Winlove CP, and Sheridan DJ. Myocardial tissue perfusion determined by par- 
ticulate and diffusible tracers during ischemia: what is measured? Cardiovasc Res 32: 869-878, 1996.

15. Folkow B. Description of the myogenic hypothesis. Circ Res 15, Suppl 1: 279-287, 1964.

16. Golino P, Piscione F, Willerson JT, Cappelli-Bigazzi M, Focaccio A, Villari B, Indolfi C, Russolillo E, Condorelli M, and Chiariello M. Divergent effects of serotonin on coronaryartery dimensions and blood flow in patients with coronary atherosclerosis and control patients. $N$ Engl J Med 324: 641648, 1991.

17. Gorman MW and Sparks HV. Progressive coronary vasoconstriction during relative ischemia in canine myocardium. Circ Res 51: 411-420, 1982.

18. Gould KL. Pressure-flow characteristics of coronary stenoses in unsedated dogs at rest and during coronary vasodilation. Circ Res 43: 242-253, 1978 .

19. Gould KL, Lipscomb K, and Calvert C. Compensatory changes of the distal coronary vascular bed during progressive coronary vasoconstriction. Circulation 51: 1085-1094, 1975.

20. Guyton RA, McClenathan JH, Newman GE, and Michaelis LL. Evolution of regional ischemia distal to a proximal coronary stenosis: self-propagation of ischemia. Am J Cardiol 40: 381$392,1977$.

21. Haljamae H. Microcirculation and hemorrhagic shock. Am $J$ Emerg Med 2: 100-107, 1984.

22. Hoffmann JIE. Determinants and prediction of transmural myocardial perfusion. Circulation 58: 381-391, 1978.

23. Klocke FJ. Coronary blood flow in man. Prog Cardiovasc Dis 19: $117-166,1976$

24. L'Abbate A, Marzilli M, Ballestra AM, Camici P, Trivella MG, Pelosi G, and Klassen GA. Opposite transmural gradients of coronary resistance and extravascular pressure in the working dog's heart. Cardiovasc Res 14: 21-29, 1980.

25. L'Abbate A, Mildenberger RR, Zborowska-Sluis DT, and Klassen GA. Myocardial tissue recruitment in the dog as determined by double tracer dilution method. Circ Res 39: 276-281, 1976.

26. Landis EM and Pappenheimer JR. Exchange of substances through capillary walls. In: Handbook of Physiology. Circulation. Bethesda, MD: Am. Physiol. Soc., 1972, sect. 2, vol. II, chapt. 29, p. 961-1034.

27. Loncar R, Flsche CW, and Deussen A. Coronary reserve of high and low flow regions in the dog heart left ventricle. Circulation 98: 262-270, 1998.

28. Marinho NVS, Keogh BE, Costa DC, Lammerstma AA, Ell PJ, and Camici PG. Pathophysiology of chronic left ventricular dysfunction. New insights from the measurement of absolute myocardial blood flow and glucose utilization. Circulation 93: 737-744, 1996.

29. Marzilli M, Sambuceti G, Fedele S, and L'Abbate A. Coronary microcirculatory vasoconstriction during ischemia in patients with unstable angina. J Am Coll Cardiol 35: 327-334, 2000

30. Maseri A, L'Abbate A, Michelassi C, Pesola A, Pisani P, Marzilli M, De Nes M, and Mancini P. Possibilities, limitations and technique for the study of regional myocardial perfusion in man by xenon-133. Cardiovasc Res 11: 277-290, 1977.

31. Miyazaki K, Hori S, Inoue S, Adachi T, Bessho M, Kuwahira I, Mori H, Nakazawa H, Aikawa N, and Ogawa S.
Characterization of energy metabolism and blood flow distribution in myocardial ischemia in hemorrhagic shock. Am J Physiol Heart Circ Physiol 273: H600-H607, 1997.

32. Pantely GA, Bristow JD, Swenson LJ, Ladley HD, Johnson WB, and Anselone CG. Incomplete coronary vasodilation during myocardial ischemia in swine. Am J Physiol Heart Circ Physiol 249: H638-H647, 1985.

33. Parodi O, Sambuceti G, Roghi A, Testa R, Inglese E, Pirelli S, Spinelli F, Campolo L, and L'Abbate A. Residual coronary reserve despite decreased resting blood flow in patients with critical coronary lesions. A study by technetium-99m human albumin microsphere myocardial scintigraphy. Circulation 87: 330-344, 1993

34. Pijls NHJ, De Bruyne B, Peels K, Van Der Voort PH, Bonnier HJ, Bartunek J, Koolen JJ, and Koolen JJ. Measurement of fractional flow reserve to assess the functional severity of coronary artery stenoses. $N$ Engl J Med 334: 17031708, 1996.

35. Rahimtoola SH. The hibernating myocardium. Am Heart $J$ 117: 211-221, 1989

36. Ronnow Sand NP, Rehling M, Bagger JP, Thuesen L, Flo C, and Nielsen TT. Functional significance of recruitable collaterals during temporary coronary occlusion evaluated by ${ }^{99 m} \mathrm{Tc}$-Sestamibi single photon emission computed tomography. J Am Coll Cardiol 35: 624-632, 2000.

37. Rubio R and Berne RM. Regulation of coronary blood flow. Prog Cardiovasc Dis 18: 105-136, 1975.

38. Sambuceti G, Marzilli M, Marraccini P, Schneider-Eicke J, Gliozheni E, Parodi O, and L'Abbate A. Coronary vasoconstriction during myocardial ischemia induced by rises in metabolic demand in patients with coronary artery disease. Circulation 95: 2652-2659, 1997.

39. Serruys PW, Di Mario C, Meneveau $\mathbf{N}$, de Jaegere $\mathbf{P}$, Strikwerda S, de Feyter PJ, and Emanuelsson H. Intracoronary pressure and flow velocity with sensor-tip guide wires: a new methodologic approach for assessment of coronary hemodynamics before and after coronary interventions. Am J Cardiol 71: 41D-53D, 1993.

40. Smith SC Jr, Gorlin R, Herman MV, Taylor WJ, and Collins JJ Jr. Myocardial blood flow in man: effects of coronary collateral circulation and coronary artery bypass surgery. J Clin Invest 51: 2556-2565, 1972.

41. Steenbergen C, Deleeuw G, Barlow C, Chance B, and Williamson JR. Heterogeneity of the hypoxic state in perfused rat heart. Circ Res 41: 606-615, 1977.

42. Taylor AE. Capillary fluid filtration. Starling forces and lymph flow. Circ Res 49: 557-575, 1981.

43. Yoshida S, Akizuki S, Gowski D, and Downey JM. Discrepancy between microsphere and diffusible tracer estimates of perfusion to ischemic myocardium. Am $J$ Physiol Heart Circ Physiol 249: H255-H264, 1985.

44. Zierler K. Equations for measuring blood flow by external monitoring of radioisotopes. Circ Res 16: 309-321, 1965.

45. Zuurbier CJ, Van Iterson $\mathbf{M}$, and Ince C. Functional heterogeneity of oxygen supply-consumption ratio in the heart. Cardiovasc Res 44: 488-497, 1999. 